\title{
Suizidales Verhalten und Suizid
}

\author{
Ulrich Hegerl ${ }^{\mathrm{a}} \quad$ Manfred M. Fichter ${ }^{\mathrm{b}}$ \\ ${ }^{a}$ Kompetenznetz Depression, Suizidalität, München, \\ ${ }^{b}$ Medizinisch-Psychosomatische Klinik Rosenek, Prien, Deutschland
}

Suizidales Verhalten ist ein einerseits schillerndes, andererseits düsteres Phänomen, durch das wir Menschen uns vom Tierreich abheben. Selbst bei Primaten wird kein suizidales Verhalten beobachtet [Eibl-Eibesfeldt, 1995]. Die Bewertung von suizidalem Verhalten und Suizid reicht von «sittlich hoch stehender Tat» bis zu «verdammenswürdiges Verhalten» oder «Sünde». Auch die Worte für die Tat belegen unsere Bewertungen: Selbsttötung, Selbstmord, Freitod. Der Leser wird folgende Selbstmorde recht unterschiedlich bewerten: Da gab es im alten Israel die Stadt Massada, deren Einwohner nicht lebend in die Hände der Römer fallen wollten und deshalb Massensuizid begingen. Da war General Rommel, der sich dem Befehl des Führers gehorchend in der Tradition soldatischer Ethik erschoss; ein anderer General (Paulus) kam in dem etwas anderen Kontext vor Stalingrad einem solchen Ansinnen des Führers nicht nach. Da war Heinrich von Kleist, der mit einer Frau einen sehr genau geplanten und inszenierten Doppelsuizid machte. Da gab es Menschen, die am 11.09.2001 im World Trade Center in New York ihrer Arbeit nachgingen bis das Flugzeug explodierte und sie vor den Flammen fliehend selbst vom Wolkenkratzer in den Abgrund sprangen. Da sind Terroristen, die sich selbst und viele andere in die Luft sprengen - oft Zivilisten. Es gibt Liebeskranke, deren Liebe nicht erwidert wird, die in ihrer Gefühlsaufwallung ihrem Leben ein vorzeitiges Ende setzen. Marilyn Monroe starb an einer Überdosis Tabletten und Hunderte machten es ihr - im Sinne des Werther-Effekts - nach. Manche schwer Krebskranke im Endstadium kommen dem natürlichen Tod um einige Tage zuvor. Geisterfahrer spielen auf der Autobahn russisches Roulette. Depressive, die nach vielen Suizidversuchen die Düsternis um sich herum nicht mehr ertragen, erhängen oder erschießen sich. Da ist der 80-Jährige, dessen Freunde und Bekannte tot sind, der seit Jahren in Einsamkeit lebt und seinem Leben durch einen Pulsaderschnitt ein Ende bereitet. - All dies lässt uns nicht kalt, es provoziert uns vielleicht. Es berührt uns. Wir bewerten es. In der Bundesrepublik Deutschland und anderen westlichen Industrieländern gehört Tod durch Suizid zu den häufigsten Todesursa- chen. Suizide sind häufiger als eine Reihe von anderen Todesursachen, die in der öffentlichen Wahrnehmung oft stärker im Vordergrund stehen (Abb. 1). Sehr viel größer ist noch die Zahl derer, die einen Suizidversuch (Parasuizid) begehen.

Auch wenn die individuelle Situation und Motivlage zum Suizidzeitpunkt völlig unterschiedlich sein kann, so ist durch mehrere psychologische Autopsiestudien recht gut belegt, dass in den westlichen Ländern die große Mehrheit der Suizide $(90 \%)$ im Rahmen psychiatrischer Erkrankungen erfolgen, am häufigsten im Rahmen einer Depression. Aus ärztlicher Sicht ist es hier unpassend, von «Freitod» zu sprechen, denn der Betroffene hat sich nicht frei für die Tat entschieden, sondern ist Opfer seiner Erkrankung geworden. Nach den epidemiologischen Statistiken sind die Suizidraten in Europa in den sonnigeren und wärmeren mediterranen Ländern wie Italien, Spanien und Griechenland oft niedriger als in nord- oder mitteleuropäischen Ländern wie Deutschland, Österreich, Dänemark, Finnland und Frankreich, allerdings weist das eher südlich gelegene Ungarn hohe, England dagegen eher niedrige Suizidraten auf. Die Nord-Süd-Differenz bei Suiziden kann auch mit der ethischen Haltung und Religionszugehörigkeit zu tun haben. Im eher protestantischen Norden ist die Suizidrate höher als im eher katholischen Süden Europas. In Europa ist bei Männern aller Altersstufen die Suizidrate höher als bei Frauen und steigt mit zunehmendem Alter an. In Deutschland hat ein alter Mann ein etwa fünffach höheres Suizidrisiko als eine gleichaltrige Frau und ein etwa zwanzigfach höheres Suizidrisiko als eine junge Frau. Suizidversuche sind dagegen bei jüngeren Frauen am häufigsten.

Die wissenschaftliche Erforschung suizidalen Verhaltens - die Suizidologie - hatte im 19. und Anfang des 20. Jahrhunderts einen Schwerpunkt auf der psychiatrisch-phänomenologischen sowie tiefenpsychologisch-psychodynamischen Betrachtung. Etwa zeitlich parallel dazu entwickelte sich die soziologisch-epidemiologische Suizidologie, deren Hauptvertreter E. Durkheim war. Postuliert wurde das Konzept des anomischen Suizids, der in Zeiten des Umbruchs und der Werteveränderung mit unzureichender Wertorientierung (Anomie) häufiger

\begin{tabular}{ll}
\hline KARGER & ๑ 2005 S. Karger GmbH, Freiburg \\
Fax +49 7614520714 & Accessible online at: \\
$\begin{array}{l}\text { E-mail Information@Karger.de } \\
\text { www.karger.com }\end{array}$ & www.karger.com/ver
\end{tabular}


auftreten sollte. Diese Konzepte berücksichtigen zu wenig die enge Verknüpfung zwischen psychiatrischer Erkrankung und Suizid und sind nahezu inkompatibel mit so manchen real beobachteten Änderungen in den Suizidraten. So kam es in den neuen Bundesländern seit 1989 zu dem stärksten Rückgang der Suizidraten in ganz Europa, trotz Werteveränderungen, sozialen Umwälzungen und Anstieg der Arbeitslosigkeit von Null auf 20\%. Im Laufe des 20. Jahrhunderts wurde es für die Psychiatrie klarer, dass Suizid in engem Zusammenhang zu psychischen Erkrankungen steht, insbesondere zu Depression, aber auch Alkoholismus und Schizophrenie. Ergänzt wird diese Sicht durch die neurobiologische Forschung in den letzten Jahrzehnten, die unter anderem genetische und neurochemische Einflüsse (z.B. «Serotoninmangel-Hypothese» bei Suizid von Marie Åsberg et al. [1976]) auf suizidales Verhalten untersuchte. Es liegt nahe, Konzepte der Suizidprävention zu entwickeln und besonders bei depressiven und anderen psychisch kranken Risikopersonen auf Ihre Wirksamkeit hin zu untersuchen. Reviews und Metaanalysen [Van der Sande et al., 1997; Hawton et al., 2001; Althaus und Hegerl, 2003] kommen hier jedoch zu dem eher ernüchternden Schluss, dass bisher keine durch empirische Daten ausreichend gestützte Suizidpräventionskonzepte vorliegen.

In den letzten Jahren wurde in Deutschland im Rahmen des vom Bundesministerium für Bildung und Forschung (BMBF) geförderten Kompetenznetzes «Depression, Suizidalität» die Forschung zu dieser Thematik vorangetrieben. Dabei gab es auch Projekte mit dem Schwerpunkt Suizidalität, bei der zahlreiche der Autoren dieses Themenschwerpunktheftes mitwirkten. Hegerl [2005] stellt in seinem Beitrag die Überlappung der Phänomene Depression und Suizidalität dar. In diesem Kontext ist das Nürnberger Bündnis gegen Depression von Interesse, im Rahmen dessen über die Optimierung der Versorgung depressiver Patienten eine deutliche Reduktion suizidaler Handlungen erzielt werden konnte, ein Konzept das zur Zeit in Kooperation mit 15 Ländern auf viele andere Regionen ausgedehnt wird. Eingegangen wird auch auf die aktuelle Diskussion zu einer möglichen Suizid induzierenden Wirkung von Antidepressiva. Der Beitrag von Althaus [2005] gibt einen Überblick über Strategien zur Suizidprävention und diskutiert kritisch die bisher unbefriedigende Evidenzlage bezüglich der Wirksamkeit der verschiedenen Maßnahmen. Ein Fazit ist, dass Interventionsstrategien am erfolgversprechendsten sind, die parallel auf verschiedenen Ebenen erfolgen (z.B. breite Öffentlichkeit, Ärzte, Psychotherapeuten, Risikogruppen) und so Synergieeffekte nutzen. Pfeiffer-Gerschel et al. [2005] thematisieren die wachsenden Chancen und Risiken, die das Internet suizidgefährdeten Patienten bietet. Manche der zahlreichen Suizidforen können über Gruppendruck, Senkung der Hemmschwelle hinsichtlich suizidaler Handlungen und Verteufelung der «Schulmedizin» negative

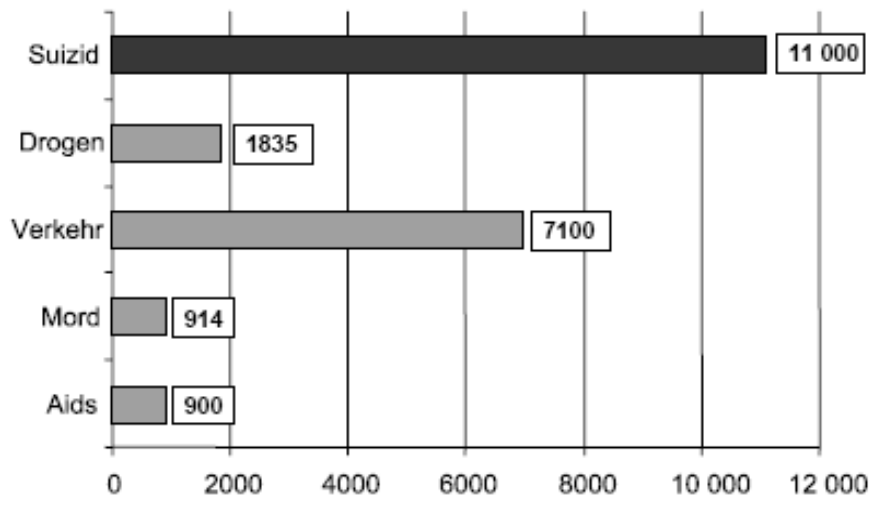

Abb. 1. Todesursachen im Vergleich: 2001. Daten des Bundesamtes für Statistik und des BMI.

Auswirkungen auf Untergruppen der Nutzer dieser Suizidforen haben. Verabredungen im Internet zum gemeinsamen Suizid und Beratung zu Suizidmethoden sowie Zusendung entsprechender tödlicher Medikamente sind weitere Facetten, die auch durch die Medien gegangen sind. Winter [2005] gibt einen Überblick über Hilfsangebote für Angehörige, die nach einem Suizid erschüttert, traumatisiert, ratlos und von Schuldgefühlen geplagt zurückbleiben. Die medikamentöse Behandlung der Suizidalität wird von Pfennig et al. [2005] dargestellt. Diese stellt nur einen Baustein im Rahmen der Krisenintervention und längerfristigen Suizidprävention dar und richtet sich je nach der zugrunde liegenden Störung. Dorrmann [2005] diskutiert kritisch den Einsatz von Non-Suizid-Verträgen. Es werden sehr konkrete Hinweise und Empfehlungen zu dieser verbreiteten, aber sehr unterschiedlich gehandhabten Intervention gegeben.

\section{Literatur}

Althaus D: Suizidprävention: Vorgehensweisen und Wirksamkeit. Verhaltenstherapie DOI: $10.1159 / 000083696$.

Althaus D, Hegerl U: The evaluation of suicide preventive activities: State of the art. World J Biol Psychiatry 2003:4:156-165.

Åsberg M, Transkman L, Thoren P: 5-HIAA in the cerebrospinal fluid: A biochemical suicide predictor? Arch Gen Psychiatry 1976;33:1193-1197.

Dorrmann W: Pro und Contra von Verträgen bei Patienten in akuten suizidalen Krisen. Verhaltenstherapie DOI: 10.1159/000082736.

Eibl-Eibesfeldt J: Die Biologie des menschlichen Verhaltens. Grundriss der Humanethologie. München, Piper, 1995.

Hawton K, Townsend E, Arensmen F, Gunnel D, Hoozell P, House A, van Heeringen K: Psychosocial and pharmacological treatments for deliberate self harm; in The Cochrane Library. Oxford, Update Software, 2001;4.

Hegerl U: Depression und Suizidalität. Verhaltenstherapie DOI: 10.1159/000083697. Pfeiffer-Gerschel T, Seidscheck I, Niedermeier N, Hegerl U: Suizid und Internet. Verhaltenstherapie DOI: 10.1159/000083679.

Pfennig A, Berghöfer A, Bauer M: Medikamentöse Behandlung der Suizidalität. Verhaltenstherapie DOI: 10.1159/000083490.

Van der Sande R, Buskens E, Allart E, van der Graaf Y, van Engeland H: Psychosocial intervention following suicide attempt: A systematic review of treatment interventions. Acta Psychiatr Scand 1997;96:43-50.

Winter S, Brockmann E, Hegerl U: Die Situation Hinterbliebener nach Suizid. Verhaltenstherapie DOI: 10.1159/000083937. 